\title{
Translational research as prerequisite for personalized psychiatry
}

\author{
Martin E. Keck ${ }^{1}$ - Nils Kappelmann ${ }^{1}$ - Johannes Kopf-Beck ${ }^{1}$
}

Published online: 15 March 2018

c) Springer-Verlag GmbH Germany, part of Springer Nature 2018

Psychiatric disorders are often conceptualized from a biopsychosocial perspective, in which factors from single genes to social environment play important etiological roles. Similarly, treatments of psychiatric disorders optimally target a range of these etiological levels, for instance, by chemical alteration (e.g., via medication) and establishment of social support structures (e.g., via psychotherapy) without being eclectic in the choice of treatment [1]. Despite such conceptual complexity, however, scientific research relies on parsimony and the formulation of targetable research hypotheses, and thus is at risk to result in a reductionist fragmentation of single-discipline approaches. Such fragmented research might produce findings that are not generalizable and difficult to translate into multidisciplinary, biopsychosocial frameworks. This stresses the need for translational research which balances the risk of being reductionist on the one hand and eclectic on the other hand, by providing treatment guidelines and establishing a personalized psychiatry.

This issue of European Archives of Psychiatry and Clinical Neuroscience provides two excellent approaches at deciphering translational components involved in substance-use disorders by linking genetic/biological markers with cognitive parameters and psychotherapy response. Zlomuzica et al. investigated how genetic predisposition towards addiction and maintenance of smoking manifests on an information-processing level. Using an approachavoidance paradigm and smoking-specific stimuli, it was shown that smokers carrying the Taq1B polymorphism of the dopamine receptor D2 (DRD2) exhibited greater tendencies to approach smoking-specific stimuli, yet avoid naturalreward stimuli [2]. Becker and colleagues used functional neuroimaging in combination with a reward sensitivity task to see whether cue exposure treatment (CET) in addition to treatment as usual (TAU) for alcohol addiction would

Martin E. Keck

keck@psych.mpg.de

1 Department of Clinical Research, Max Planck Institute of Psychiatry, Kraepelinstr. 2-10, 80804 Munich, Germany lead to changes in brain activation when compared to TAU only. Results demonstrated increased prefrontal cortex activation in superior frontal gyrus and anterior cingulate cortex in individuals with high-baseline reward sensitivity following additional CET. These activation changes were related to increased self-efficacy for achieving abstinence and a decreased temptation to drink, which highlights that baseline reward sensitivity might serve as prerequisite for successful CET [3]. Both of these studies highlight the necessity of a multivariate, biopsychosocial perspective for disorder conceptualization: only by taking multidisciplinary information, such as relevant genotypes (e.g., DRD2 Taq1B polymorphism) or reward sensitivity, into account for treatment selection, successfully individualized treatment can be assured.

Another challenge for the understanding and treatment of psychiatric disorders are comorbidities, which can influence the disorder course on all levels of the biopsychosocial model. For instance, the new S3 guidelines presented by Preuss et al. highlight how diagnostics and treatment of alcohol use disorder depend on the presence and type of comorbidities such as affective disorders or schizophrenic psychoses. The interdisciplinary and evidence-based guidelines illustrate the necessity to include the symptom context as well as different levels of therapy such as pharmaco-, psychotherapy and psychosocial interventions [4]. Schirmbeck et al. examined the impact of co-occurring obsessive-compulsive symptoms (OCS) on clinical characteristics in schizophrenia. Results indicate that patients with comorbid obsessive-compulsive symptoms suffer from more severe psychotic and affective symptomatology, which, in turn, leads to lower functioning and higher psychopathological burden. Furthermore, the authors found an association of symptom remission, suggesting that OCS, psychotic and affective symptoms functionally effect each other [5]. These two studies thus emphasize the importance of considering and integrating comorbidities in psychiatric research and, ultimately, clinical practice.

The complexity of the field imposes requirements for translational psychiatry regarding research designs and data 
collection, the use of multivariate data analysis, and finally, the implementation of a personalized psychiatry in clinical routine processes.

Research designs should move beyond single disorder assessments and understand symptoms as interdependent phenomena, rather than categorical diagnostic entities. A concept of disorders as networks of symptoms which reciprocally effect each other gains its clinical relevance and explanatory power by including data from different sources, i.e. biological/genetic data, cognitive functioning, behavioral parameters, i.e. locomotor activity or sleep habits, but also self-reports on psychologically relevant domains, i.e. biographically relevant life events, experiencing and coping with certain emotions. Considering these networks as dynamic (in a temporal sense) also visualizes the need for a data collection at multiple time points. Progress in the development of technical advices in the recent years in fields such as actigraphy, and application-based self-reports facilitates approaches such as ecological momentary assessment and enables new opportunities in therapy process research. Such comprehensive multi-level data collection requires the statistical and methodological tools to analyze such multivariate systems.

The establishment of Research Domain Criteria (RDoC) as an additional perspective upon scientific studies has further promoted the inclusion of translational assessments in distinct functional domains such as valence, cognitive, and social processes that are independent from traditional psychiatric taxonomy [6]. Although the RDoC perspective is highly optimistic in its assumption that we are able to visualize psychopathological processes with current tools of clinical neuroscience, this outlook forces researchers to challenge themselves and develop new methodologies. One important step towards this goal will be to make sense of the high-dimensional data generated in translational, multidisciplinary studies. The recently developed network theory of mental disorders, in combination with network analysis techniques, offers one approach for interpreting multi-causal systems [7]. In contrast to the traditional common cause model, network theory treats symptoms as independent- yet interacting-constructs that can each have distinct, underlying etiologies. This allows visualizing potential causal pathways between symptoms transdiagnostically, so comorbidities are a natural part of these networks.

Despite these advantages of network models, however, these analyses still have complexity restrictions as their robustness is dependent upon sample size, number of measurement points, and the number of variables included in the network [8]. Thus, an important prerequisite for targeting translational research hypotheses using network analysis will be advances in other methodologies that allow meaningful reduction of the number of variables. Here, computational psychiatry offers tools to reduce dimensionality in a data-driven (e.g., unsupervised machine learning) or theorydriven (e.g., Bayes optimal modelling) way [9]. Complemented by other validated integration approaches such as polygenic risk score computation, these techniques might enable researchers to extract the gist from high-dimensional translational data, which could, in turn, be utilized to make generalizable between-subject and within-subject inferences from network models and predict therapeutic trajectories on an individual basis.

Moving the focus from diagnostics to interventions, the insights gained from multivariate measurements and data analyses have to find their way into the therapeutic context.

Thus, the application of new knowledge in clinical routine processes itself should be the object of research. Personalized psychiatry should not be restricted to individualized patient profiles and risk scores, allowing for the assignment of a specific therapeutic intervention, but extended to a rather dynamic understanding. Based on the idea of therapy as a process of change, continual monitoring of the individual symptom course on different levels and dimensions facilitates consequential re-adjustment of therapeutic interventions.

At the Max Planck Institute of Psychiatry, there is a current approach to address design and methodological challenges in a large-scale randomized, controlled study evaluating three different psychotherapeutic methods as treatment of major or persistent depressive disorder (clinicaltrials.gov ID: NCT03287362). Beyond general efficacy investigation and comparison of schema therapy, cognitive behavioral therapy, and individual supportive therapy, a vast array of biological (e.g., genetic, epigenetic, inflammatory), brain imaging, social, cognitive, psychometric, and (neuro-) psychological information on patients is collected, aiming to identify optimal patient-therapy combinations for future personalized treatment. Moreover, the RCT-trial is conducted in an inpatient and day clinic setting and incorporated into usual psychiatric care while minimizing restraints on inclusion criteria such as psychiatric comorbidities. With high-dimensional translational data, and state-of-the-art methodologies and analyses, the study should provide a general framework from which answers to hypotheses of psychiatric research can be deduced.

Combining translational, multidisciplinary research designs with recent advances in techniques of data collection and multivariate analysis methods is promising in leading to new insights on psychiatric taxonomy in line with $\mathrm{RDoC}$, network theoretical perspectives, and time line analysis regarding the course of symptoms over time. This may potentially surpass unitary diagnostic categories but could allow coverage of comorbidities in conjunction with an understanding of fundamental biological processes. Finally, it could pave the way toward a paradigm shift, in which we are able to harness an evidence-based, multidisciplinary 
knowledge to treat patients based on a truly personalized treatment plan.

\section{References}

1. Nassir Ghaemi S (2009) The rise and fall of the biopsychosocial model. Br J Psychiatry 195:3-4. https://doi.org/10.1192/bjp. bp.109.063859

2. Zlomuzica A, Machulska A, Roberts S et al (2018) The dopamine D2 receptor mediates approach-avoidance tendencies in smokers. Eur Arch Psychiatry Clin Neurosci. https://doi.org/10.1007/s0040 6-017-0793-y

3. Becker A, Gerchen MF, Kirsch M et al (2018) Striatal reward sensitivity predicts therapy-related neural changes in alcohol addiction. Eur Arch Psychiatry Clin Neurosci. https://doi.org/10.1007/ s00406-017-0805-y

4. Preuss UW, Gouzoulis-Mayfrank E, Havemann-Reinecke U et al (2018) Psychiatric comorbidity in alcohol use disorders: results from the German S3 guidelines. Eur Arch Psychiatry Clin Neurosci. https://doi.org/10.1007/s00406-017-0801-2

5. Schirmbeck F, Swets M, Meijer CJ et al (2018) Obsessive-compulsive symptoms and overall psychopathology in psychotic disorders: longitudinal assessment of patients and siblings. Eur Arch Psychiatry Clin Neurosci. https://doi.org/10.1007/s0040 6-016-0751-0

6. Insel T, Cuthbert B, Garvey M et al (2010) Research Domain Criteria $(\mathrm{RDoC})$ : toward a new classification framework for research on mental disorders. Am J Psychiatry 167:748-751. https://doi. org/10.1176/appi.ajp.2010.09091379

7. Borsboom D (2016) A network theory of mental disorders. World Psychiatry 16:5-13. https://doi.org/10.1002/wps.20375

8. Epskamp S, Borsboom D, Fried EI (2018) Estimating psychological networks and their accuracy: a tutorial paper. Behav Res 50:195-212. https://doi.org/10.3758/s13428-017-0862-1

9. Huys QJM, Maia TV, Frank MJ (2016) Computational psychiatry as a bridge from neuroscience to clinical applications. Nat Neurosci 19:404-413. https://doi.org/10.1038/nn.4238 